\title{
Further Study on the Evaluation of Molecular Weight Dependence of $\chi$-Parameter by Phase Equilibrium Experiment on Polystyrene-Methylcyclohexane System
}

\author{
Kenji Kamide, Tatsuyuki ABE, and Yukio MiYaZAKI \\ Textile Research Laboratory, Asahi Chemical Ind. Co. Ltd., \\ Takatsuki, Osaka 569, Japan.
}

(Received November 13, 1981)

\begin{abstract}
For evaluating the molecular weight dependence of a thermodynamic interaction parameter $\chi$, phase equilibrium experiments on the polystyrene-methylcyclohexane system were carefully carried out. The mathematical phase equilibrium of this polymer-solvent system was determined by taking into account the molecular weight- and concentration-dependences of the $\chi$ parameter, according to the expression $\chi=\chi_{00}(1+k / X)\left(1+p v_{\mathrm{p}}\right)$, where $\chi_{00}$ is a constant independent of both polymer molecular weight and concentration, $X$, the molar volume ratio of the polymer to the solvent, $v_{\mathrm{p}}$, the polymer volume fraction, and $k$ and $p$ are molecular weight- and concentration-dependence coefficients. The partition coefficient $\sigma$ increased remarkably with $1 / X$ and $k$ decreased linearly with an increase in the phase equilibrium temperature $T$, generally expressed by $k=k_{0}(1-T / \theta)\left(\theta\right.$ is Flory's theta temperature and $k_{0}$ is a constant).

KEY WORDS Phase Equilibrium / Thermodynamic Interaction Parameter/

Polystyrene / Partition Coefficient /
\end{abstract}

In a previous paper, ${ }^{1}$ we established a generalized theory for describing the phase equilibrium of multi-component polymer-single solvent systems, by introducing the molecular weight- and concentration-dependences of the polymer-solvent thermodynamic interaction parameter $\chi$ into the well-known Flory-Huggins-Fujishiro theory. The $\chi$-parameter is expressed in the form:

$$
\chi=\chi_{00}(1+k / X)\left(1+p v_{\mathrm{p}}\right)
$$

where $\chi_{00}$ is a constant independent of both polymer molecular weight and concentration, $X$, the ratio of the molar volume of the polymer to that of the solvent, $v_{\mathrm{p}}$, the polymer volume fraction, and $k$ and $p$ are molecular weight- and concentrationdependence coefficients, respectively. It should be noticed that eq 1 is a semi-empirical equation and the $k$ and $p$-parameters are phenomenological coefficients, although essentially the same expression for $\chi$ as a function of $X$ and $v_{\mathrm{p}}$ can be readily derived from the refined lattice theory which has a severe limit in applicability to polymer solutions. ${ }^{1}$ In eq 1 , it is assumed that the $k$-parameter is independent of the polymer volume fraction and the $p$-parameter is independent of molecular weight. This assumption is supported by the following experimental facts: (1) The unique $k$-temperature relation has been obtained, regardless of $v_{\mathrm{p}}$, for the polystyrene (PS)cyclohexane $(\mathrm{CH})$ system (Figure 4 of ref 1 ). A similar observation will be presented in a later section of this paper (see Figure 5). (2) In the strict sense, the $\chi$-parameter is not a linear function of $v_{\mathrm{p}}$ but depends also on higher powers of $v_{\mathrm{p}}$. But, in the $v_{\mathrm{p}}$ range of phase equilibrium experiments on polymer solutions $\left(0 \leq v_{\mathrm{p}} \leq 0.5\right), \chi$ is reasonably linearly dependent on $v_{\mathrm{p}}$ (for example, Figure 1 of ref 2 for the PS-methylcyclohexane (MCH) system). (3) When evaluated from fractionation data on molecular weight distribution (or the molecular weight distribution parameter), the polymer volume fraction in the polymer-rich phase $v_{\mathrm{p}(2)}$ and the volume ratio of the polymer-lean to the polymer-rich phase $R$, by comparison with the corresponding theoretical values, the values of $p$ coincide well with each other, and are almost constant throughout the fractionation run. ${ }^{3,4}$ This strongly implies that a first approximation the $p$-parameter may be considered as constant throughout temperature and 
average molecular weight ranges employed in the fractionation. In addition, the reliability of $p$ estimated from a phase separation study has been confirmed by relevant literature data for the PS- $\mathrm{CH}$ system ${ }^{4}$ and also by our experimental data on $p$ for the PS-MCH system ${ }^{2}$ determined on the basis of other principles, including osmotic pressure, isothermal distillation, vapor pressure, critical miscibility and ultracentrifuge (Table $\mathrm{V}$ of ref 3 and Table III of ref 4), in spite of the appreciable differences in average molecular weight and molecular weight distribution of the polymer samples employed. As is evident from the above, eq 1 is sufficiently accurate for describing phase separation phenomena of polydisperse polymer-solvent systems.

The molecular weight dependence of the $\chi$ parameter is closely related to the molecular weightdependence of the partition coefficient $\sigma$ of a polymer having $X$ between the polymer-rich and polymer-lean phases (see eq 3 ).

Using mathematical calculations based on the above theory, we have made a careful study of the effects of the $k$ - and $p$-parameters on the phase equilibrium and the molecular weight fractionation characteristics of multi-component polymer-single solvent systems. ${ }^{1}$ We found that the positive molecular weight dependence of the $\chi$-parameter $(k>0)$ gives rise to a larger polydispersity of the polymers in the polymer-rich and lean-phases, particularly when $k>3$. In the region of low $\rho_{\mathrm{p}}\left(\rho_{\mathrm{p}}\right.$ is the weight fraction of the polymer in a polymer-rich-phase relative to the total polymer dissolved), the ratio of the weight- to number-average of $X, X_{w} / X_{n}$, in the polymer-rich phase is remarkably influenced by both $p$ and $k$. In the region of high $\rho_{\mathrm{p}}$, the polydispersity of the polymer in the polymer-lean phase is predominantly controlled by $k$. For PS- $\mathrm{CH}$ and PS$\mathrm{MCH}$ systems, $k$ was found by the following means to lie between 0-10: (1) Comparison of the theoretical and experimental relations between $\sigma$ and $1 / X,(2)$ osmometric determination of $\chi$ as a function of molecular weight and concentration, and (3) comparison of molecular weight distributions (MWD) of the polymer in the two phases with the theoretical curves calculated assuming various values for $k$. The tendency for $k$ to decrease with an increase in the phase equilibrium temperature, independently of the concentration, was clearly observed. It was also found for the PS-CH system that $k$ is nearly zero at Flory's theta temperature. Unfortunately, the molecular weight range, in which $\sigma$ was examined in the previous paper, ${ }^{1}$ was too narrow to determine the temperature dependence of $k$ accurately.

In this article we have attempted to carry out further study on the phase equilibrium of the PS$\mathrm{MCH}$ system over a sufficiently wide range of $X$ $(600 \leq X \leq 5000)$ and determine the molecular weight dependence of $X$ as a function of temperature. For this purpose, the MWD of polymers partitioned in both phases were evaluated very carefully by high performance liquid chromatography (HPLC) using columns with 12000 theoretical plates each $50 \mathrm{~cm}$ in length.

\section{EXPERIMENTAL}

\section{Polymer}

The same PS sample as that used in previous papers ${ }^{3-5}$ was employed here and was characterized as follows: the weight-average molecular weight $M_{w}$ was $23.9 \times 10^{4}$ by gel permeation chromatography (GPC) in tetrahydrofurane (THF) and $23.2 \times 10^{4}$ by light scattering in benzene at $25^{\circ} \mathrm{C}$ and a manual extrapolation of the Zimm plot, the numberaverage molecular weight $M_{n}$ was $8.6 \times 10^{4}$ by GPC in THF and $8.9 \times 10^{4}$ by membrane osmometry in toluene at $25^{\circ} \mathrm{C}$.

This polymer was independently characterized by a polymer group at Essex University (England) using a light scattering technique ${ }^{6}: M_{w}=23.3 \times 10^{4}$ by computer analysis program I, $23.8 \times 10^{4}$ by

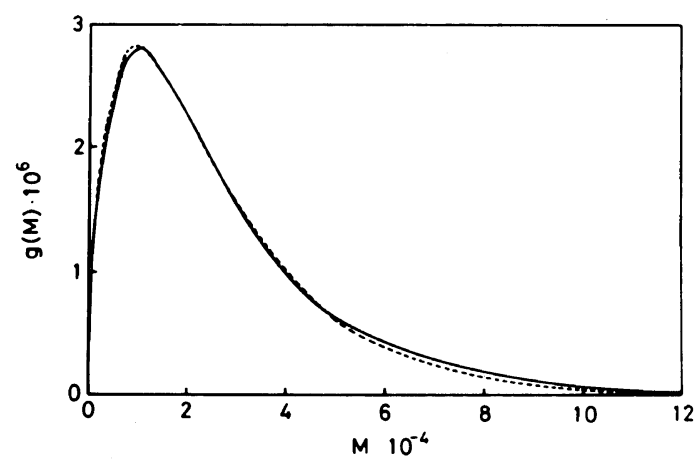

Figure 1. Molecular weight distribution curve $g(X)$ of the polymer sample used for the phase separation experiment: full line, experimental curve; broken line, SchulzZimm distribution curve with $M_{w}=24.0 \times 10^{4}$ and $M_{w} /$ $M_{n}=2.8$. 
Molecular Weight Dependence of $\chi$-Parameter

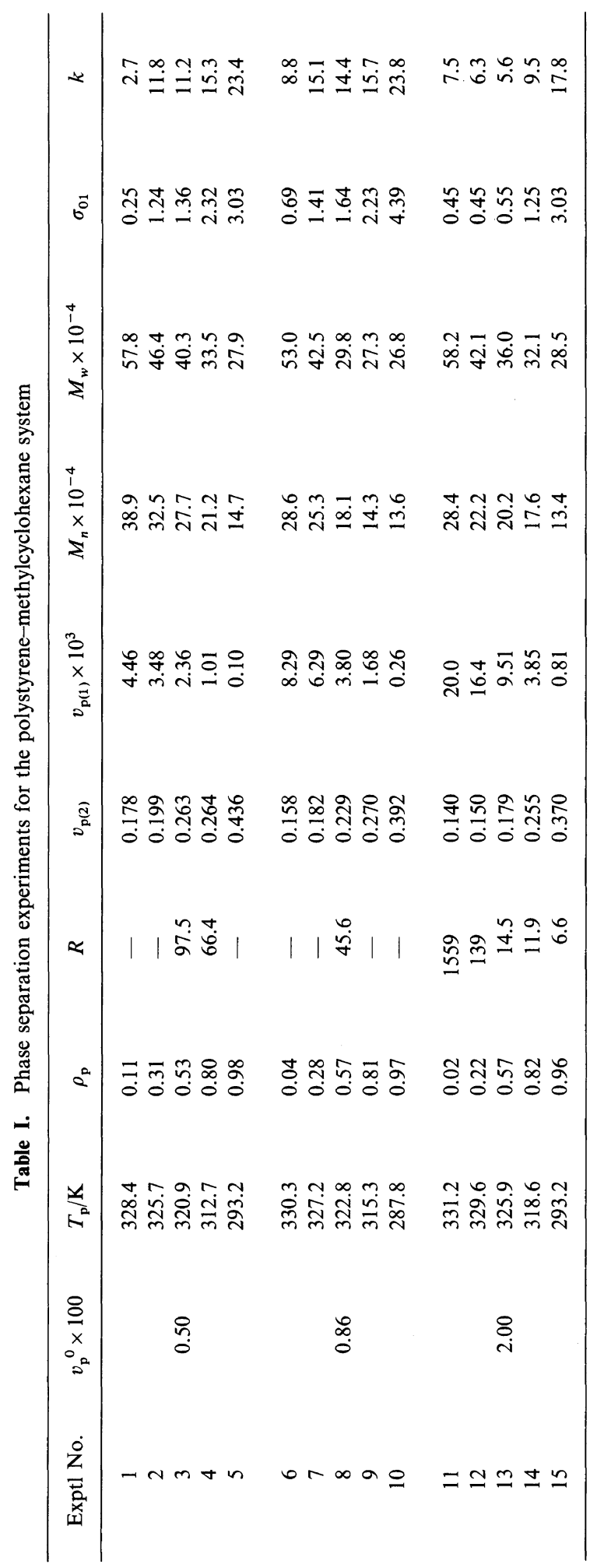


computer analysis program II and $22.0 \times 10^{4}$ by computer analysis program III, nonlinear leastsquare program. The $M_{w}$ values obtained by two computer programs based on two different models for regression analysis are in good agreement with those evaluated by us.

The MWD of the polymer estimated by HPLC was accurately represented by the Schulz-Zimm distribution with $M_{w}=24.0 \times 10^{4}$ and $M_{w} / M_{n}=2.8$, as shown in Figure 1.

\section{Solvent}

Reagent grade $\mathrm{MCH}$ and THF were distilled immediately prior to use.

\section{Phase Equilibrium Experiments}

The PS sample was dissolved in $\mathrm{MCH}$ at $70^{\circ} \mathrm{C}$ to obtain starting solutions. The phase equilibrium experiments were performed accoridng to the procedure described before. ${ }^{3,4}$ The experimental conditions, such as initial polymer volume fraction $v_{\mathrm{p}}{ }^{0}$, and phase equilibrium temperature $T_{\mathrm{p}}(\mathrm{K})$, are summarized in the second to fourth columns of Table I. The weight fraction of the polymer in the polymerrich phase (i.e., fraction size), $\rho_{\mathrm{p}}$, the volume ratio of the polymer-lean phase to the polymer-rich phase, $R$, the polymer volume fraction in the polymer-rich phase, $v_{\mathrm{p}(2)}$ and that of the polymer-lean phase, $v_{\mathrm{p}(1)}$ were determined in the same manner as described before. ${ }^{3}$

\section{Molecular Weight Distributions of Polymers in the}

\section{Two Phases}

Molecular weight distributions (MWD) for polymers separated by the phase equilibrium experiments were determined on a Shimadzu high performance liquid chromatograph model LC-1. The values of $M_{w}$ and $M_{n}$ were calculated from the MWD curves obtained. Crosslinked PS (HSG, manufactured by Shimadzu Ltd.) was used as the gel for fractionation. Two columns $(50 \mathrm{~cm}$ in length) packed with HSG-30 and HSG-50 were connected in series. A theoretical plate number of the columns was $12000 / 50 \mathrm{~cm}$ for acetone at a $1.0 \mathrm{~cm}^{3} \mathrm{~min}^{-1}$ flow rate. This instrument was operated using THF as the solvent at $25^{\circ} \mathrm{C}$ under the following conditions: Concentration of solution, $1.0 \mathrm{wt} \%$; flow rate, $1.0 \mathrm{~cm}^{3} \mathrm{~min}^{-1}$; and injection volume, $20 \mu \mathrm{l}$; fifteen samples of well-characterized living PS supplied by Pressure Chemical Co. ranging $M_{w}$ from
600 to $2 \times 10^{6}$ were used as references. Yau and Fleming's method ${ }^{7}$ was employed in order to transform the GPC curve to MWD curve. The plot of log $M_{w}$ vs. elution volume $V\left(\mathrm{~cm}^{3}\right)$ is accurately represented by,

$$
\begin{aligned}
\log M_{w}= & 24.26 V^{3}-1.89_{2} V^{2} \\
& +6.44 \times 10^{-2} V-7.89 \times 10^{-4}
\end{aligned}
$$

\section{Partition Coefficient}

We denote the heights in the normalized MWD curve $(g(X))$ at $X$ of the polymers in the polymerrich and -lean phases, by $g_{(2)}(X)$ and $g_{(1)}(X)$, respectively. The partition coefficient $\sigma$ at $X$, designated by $\sigma$ to avoid complication, is defined

$$
\sigma=(1 / X) \ln \left\{\left(v_{\mathbf{p}(2)} \cdot g_{(2)}(X)\right) /\left(v_{\mathbf{p}(1)} \cdot g_{(1)}(X)\right)\right\}
$$

In the previous paper ${ }^{1}$ on the basis of modified Flory-Huggins theory and eq 1 , we derived the relation,

$$
\sigma=\sigma_{0}+\sigma_{01} / X
$$

with,

$$
\begin{aligned}
\sigma_{0}= & \left\{\ln \frac{\left(1-v_{\mathbf{p}(1)}\right)}{\left(1-v_{\mathbf{p}(2)}\right)}+\left(v_{\mathbf{p}(2)}-v_{\mathbf{p}(1)}\right)+\left(\frac{v_{\mathbf{p}(2)}}{X_{n(2)}}-\frac{v_{\mathbf{p}(1)}}{X_{n(1)}}\right)\right\} \\
& \times\left[2\left(v_{\mathbf{p}(2)}-v_{\mathbf{p}(1)}\right)+1.5 p\left(v_{\mathbf{p}(2)}^{2}-v_{\mathbf{p}(1)}^{2}\right)\right. \\
& \left.+k\left\{\left(\frac{v_{\mathbf{p}(2)}}{X_{n(2)}}-\frac{v_{\mathbf{p}(1)}}{X_{n(1)}}\right)+p\left(\frac{v_{\mathbf{p}(2)}^{2}}{X_{n(2)}}-\frac{v_{\mathbf{p}(1)}^{2}}{X_{n(1)}}\right)\right\}\right] / \\
& \left.+k\left\{\left(\frac{v_{\mathbf{p}(2)}^{2}}{X_{n(2)}}-\frac{v_{\mathbf{p}(1)}^{2}}{X_{n(1)}}\right)+p\left(\frac{v_{\mathbf{p}(2)}^{3}}{X_{n(2)}}-\frac{v_{\mathbf{p}(1)}^{3}}{X_{n(1)}}\right)\right\}\right] \\
& -\ln \frac{\left(1-v_{\mathbf{p}(1)}\right)}{\left(1-v_{\mathbf{p}(2)}\right)}
\end{aligned}
$$

and

$$
\begin{aligned}
\sigma_{01}= & k\left\{\ln \frac{\left(1-v_{\mathrm{p}(1)}\right)}{\left(1-v_{\mathrm{p}(2)}\right)}+\left(v_{\mathrm{p}(2)}-v_{\mathrm{p}(1)}\right)+\left(\frac{v_{\mathrm{p}(2)}}{X_{n(2)}}-\frac{v_{\mathrm{p}(1)}}{X_{n(1)}}\right)\right\} \\
& \times\left\{\left(v_{\mathrm{p}(2)}-v_{\mathrm{p}(1)}\right)+0.5 p\left(v_{\mathrm{p}(2)}^{2}-v_{\mathrm{p}(1)}^{2}\right)\right\} \\
& {\left[\left(v_{\mathrm{p}(2)}^{2}-v_{\mathrm{p}(1)}^{2}\right)+p\left(v_{\mathrm{p}(2)}^{3}-v_{\mathrm{p}(1)}^{3}\right)\right.} \\
& \left.+k\left\{\left(\frac{v_{\mathrm{p}(2)}^{2}}{X_{n(2)}}-\frac{v_{\mathrm{p}(1)}^{2}}{X_{n(1)}}\right)+p\left(\frac{v_{\mathrm{p}(2)}^{3}}{X_{n(2)}}-\frac{v_{\mathrm{p}(1)}^{3}}{X_{n(1)}}\right)\right\}\right]
\end{aligned}
$$




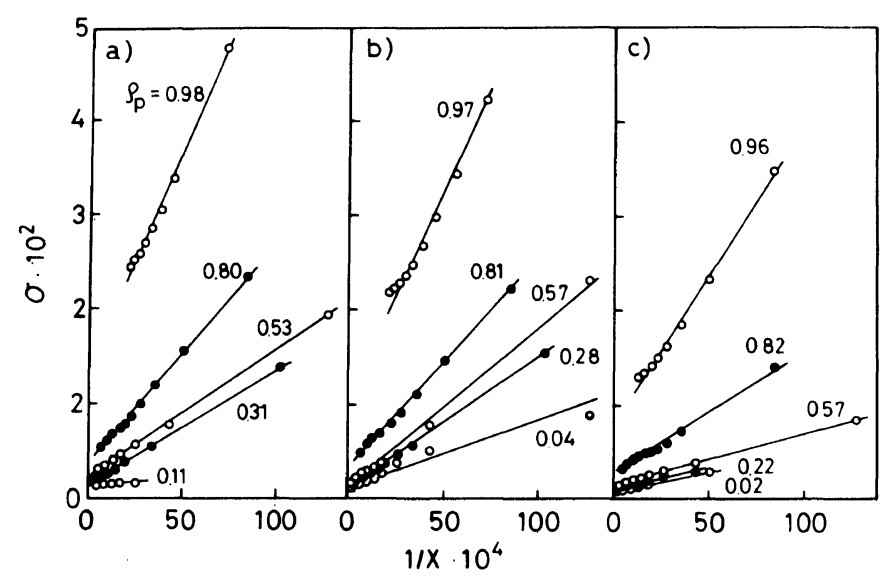

Figure 2. Molecular weight dependence ( $X=$ polymer-to-solvent molar volume ratio) of the partition coefficient $\sigma$ for polystyrene-methylcyclohexane system: a) initial polymer volume fraction $v_{\mathrm{p}}{ }^{0}=$ $0.50 \times 10^{-2}$, b) $v_{\mathrm{p}}{ }^{0}=0.86 \times 10^{-2}$, and c) $v_{\mathrm{p}}{ }^{0}=2.0 \times 10^{-2}$. Numbers on curve are the relative weight fractions $\rho_{\mathrm{p}}$ of polymer in the polymer-rich phase.

\section{Computer Simulation}

The mathematical phase equilibrium was undertaken according to the simulative procedure described in the previous paper. ${ }^{1}$ The original PS sample was assumed to have a Schulz-Zimm distribution with $X_{w}=1810$ and $X_{w} / X_{n}=2.8$. The $p$ parameter in eq 1 was experimentally found to be 0.7 by the phase equilibrium, ${ }^{3} 0.78$ by the osmotic pressure, ${ }^{7}$ and 0.6 by the cloud point method. ${ }^{8}$ In this study, $p$ was taken to be 0.7 . The parameter $k$ was varied in the range $0-20$. Other parameters including $v_{\mathrm{p}}{ }^{0}$ and $\rho_{\mathrm{p}}$ were adapted to the values selected in the actual experiments.

\section{RESULTS AND DISCUSSION}

The fifth to ninth columns of Table I show the experimental results for the volume ratio $R$, the polymer-volume fractions in the two phases, $v_{\mathrm{p}(2)}$ and $v_{\mathrm{p}(1)}$, and $M_{w}$ and $M_{n}$ of the polymer in the polymer-rich phase. Figures $2 \mathrm{a}-\mathrm{c}$ ) show empirical relationship between $\sigma$ and $1 / X$ for the PS/MCH system. Throughout the range of $v_{\mathrm{p}}{ }^{0}$ investigated, the plot can be reasonably represented by a straight line, whose slope is equal to $\sigma_{01}$ defined in eq 4. $\sigma_{01}$ increases with an increase in $\rho_{\mathbf{p}}$ (i.e., with a decrease in $T_{\mathrm{p}}$ ). The $\sigma_{01}$ values are positive and summarized in the tenth column of Table I. As is evident from the table, the partition coefficient $\sigma$ depends on the molecular weight.

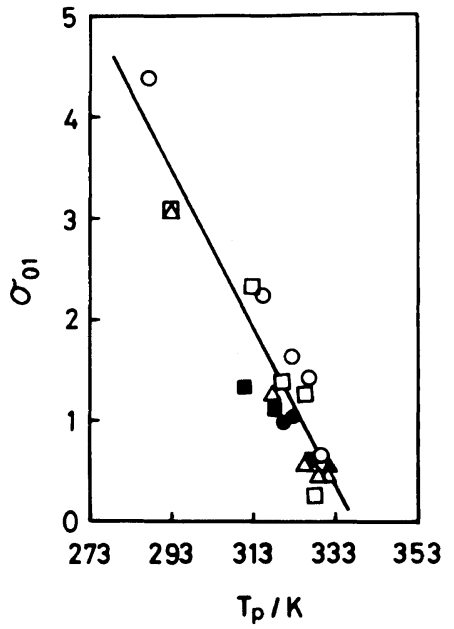

Figure 3. Variation in the parameter $\sigma_{01}$ in eq 6 with the phase separation temperature $T_{\mathrm{p}}$; rectangle, initial polymer volume fraction $v_{\mathrm{p}}{ }^{0}=0.50 \times 10^{-2}$; circle, $v_{\mathrm{p}}{ }^{0}=$ $0.86 \times 10^{-2}$; triangle, $2.0 \times 10^{-2}$; open mark, this work; closed mark, data of ref 1 ; full line, the regression line given by eq 7 .

Figure 3 shows the variation in $\sigma_{01}$ with the phase equilibrium temperature $T_{\mathrm{p}}$ for the PS-MCH system. The figure includes our previous data for the PS-MCH system. ${ }^{1}$ In the previous paper, ${ }^{1}$ only a few points were available for larger $\rho_{\mathrm{p}}$ in establishing $\sigma v s$. the $1 / X$ plot, from which $\sigma_{01}$ was estimated. The previous data for $\rho_{\mathrm{p}} \geq 0.88$ were excluded be- 
cause of the considerable uncertainty involved in the estimation of $\sigma_{01}$. It can be seen from Figure 3 that the molecular weight dependence of the partition coefficient is a unique function of the temperature, independent of concentration.

The following relation was obtained by the leastsquare method,

$$
\begin{gathered}
\sigma_{01}=25.3-0.75 T_{\mathrm{p}} \quad \text { for } \quad \text { PS-MCH }, \\
290 \mathrm{~K} \leq T_{\mathrm{p}}(\mathrm{K}) \leq 350 \mathrm{~K}
\end{gathered}
$$

The theoretical relations between $k$ and $\sigma_{01}$, calculated by computer simulation under the same conditions as for actual phase equilibrium experiments, are shown in Figure 4. Using these relations and experimental $\sigma_{01}$ data in Table I, we were able to evaluate the magnitude of $k$, and the results are listed in the last column of Table I.

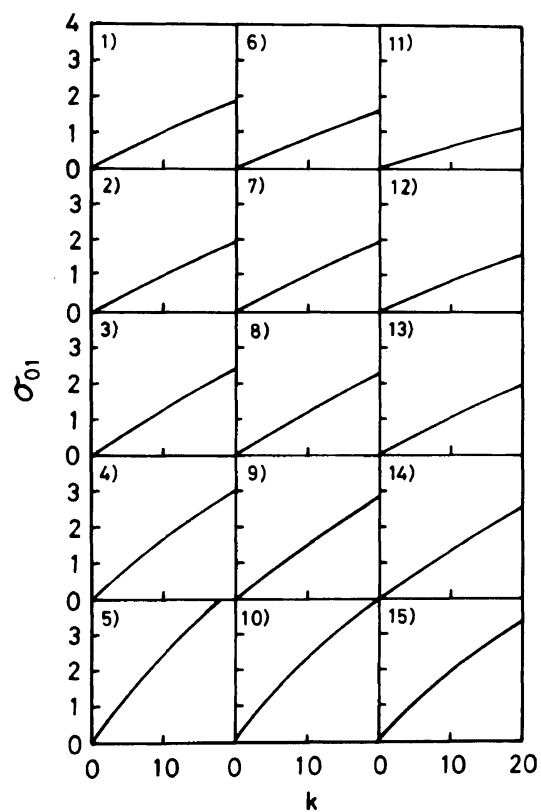

Figure 4. Theoretical relationships between $\sigma_{01}$ and the $k$-parameter: the original polymer (Schulz-Zimm distribution, $\left.X_{w}=1810, X_{w} / X_{n}=2.8\right), p=0.7$ : 1) $v_{\mathrm{p}}{ }^{0}=$ $0.50 \times 10^{-2}, \rho_{\mathrm{p}}=0.11$; 2) $v_{\mathrm{p}}^{0}=0.50 \times 10^{-2}, \rho_{\mathrm{p}}=0.31$; 3) $\left.v_{\mathrm{p}}{ }^{0}=0.50 \times 10^{-2}, \rho_{\mathrm{p}}=0.53 ; 4\right) v_{\mathrm{p}}{ }^{0}=0.50 \times 10^{-2}, \rho_{\mathrm{p}}=$ $\left.0.80 ; 5) v_{\mathrm{p}}^{0}=0.50 \times 10^{-2}, \rho_{\mathrm{p}}=0.98 ; 6\right) v_{\mathrm{p}}^{0}=0.86 \times 10^{-2}$, $\rho_{\mathrm{p}}=0.04 ;$ 7) $\left.\quad v_{\mathrm{p}}{ }^{0}=0.86 \times 10^{-2}, \quad \rho_{\mathrm{p}}=0.28 ; 8\right) \quad v_{\mathrm{p}}{ }^{0}=$ $0.86 \times 10^{-2}, \rho_{\mathrm{p}}=0.57$; 9) $\left.v_{\mathrm{p}}{ }^{0}=0.86 \times 10^{-2}, \rho_{\mathrm{p}}=0.81 ; 10\right)$ $v_{\mathrm{p}}{ }^{0}=0.86 \times 10^{-2}, \rho_{\mathrm{p}}=0.97$; 11) $v_{\mathrm{p}}{ }^{0}=2.0 \times 10^{-2}, \rho_{\mathrm{p}}=$ 0.02 ; 12) $v_{\mathrm{p}}^{0}=2.0 \times 10^{-2}, \rho_{\mathrm{p}}=0.22$; 13) $v_{\mathrm{p}}{ }^{0}=2.0 \times 10^{-2}$ ， $\rho_{\mathrm{p}}=0.57 ;$ 14) $\left.\quad v_{\mathrm{p}}^{0}=2.0 \times 10^{-2}, \quad \rho_{\mathrm{p}}=0.82 ; 15\right) \quad v_{\mathrm{p}}{ }^{0}=$ $2.0 \times 10^{-2}, \rho_{\mathrm{p}}=0.96$.

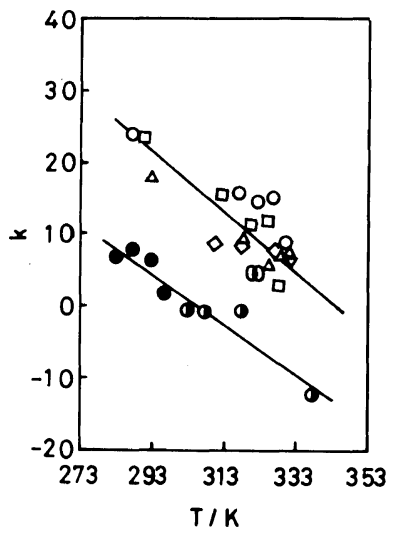

Figure 5. Changes in the $k$-parameter with temperature $T$ : closed and half-closed mark, PS/CH system; (Kamide-Miyazaki ${ }^{1}$ ), $\mathbf{\text { , }}$ (Scholte by light scattering); ${ }^{9,10}$ open mark, PS/MCH system; $\square, v_{\mathrm{p}}{ }^{0}=0.50 \times 10^{-2}$; $\diamond, \quad v_{\mathrm{p}}{ }^{0}=0.47 \times 10^{-2} ; \quad \bigcirc, \quad v_{\mathrm{p}}{ }^{0}=0.86 \times 10^{-2} ; \quad 0, \quad v_{\mathrm{p}}{ }^{0}=$ $0.94 \times 10^{-2} ; \triangle, v_{\mathrm{p}}{ }^{0}=2.0 \times 10^{-2} ; \nabla, v_{\mathrm{p}}{ }^{0}=1.86 \times 10^{-2}$.

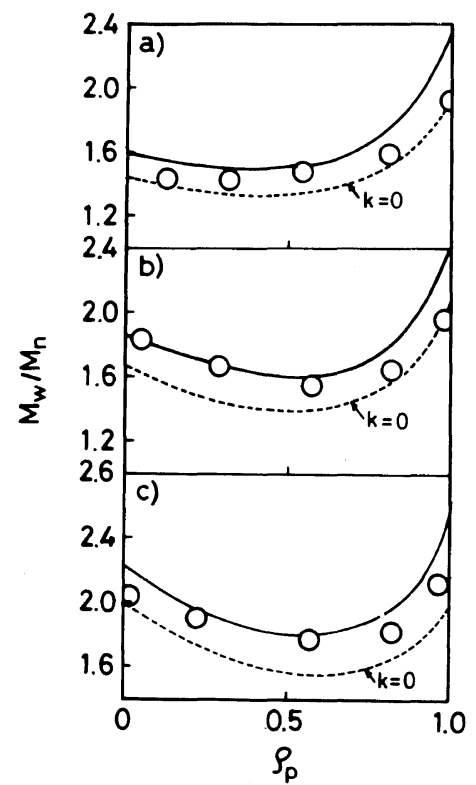

Figure 6. Dependence of the ratio $M_{w} / M_{n}$ of polymer in the polymer-rich phase on the relative amount of the polymer (i.e., the fraction size) $\rho_{\mathrm{p}}$ : open circle, experimental data points for PS/MCH system; full line, theoretical curve calculated under the same conditions as those in actual experiments (in this case, temperature dependence of $k$ (see Figure 5) is taken into account); broken line, theoretical curve calculated by assuming $k=0$ under the same conditions as those in actual experiments, except for $k$. 
Figure 5 shows the temperature dependence of $k$ for the PS-MCH system. In this figure, our previous data for PS- $\mathrm{MCH}^{1}$ except for $\rho_{\mathrm{p}} \geq 0.88$ and those for PS- $\mathrm{CH}^{1}$ are shown for comparison. The $k$ parameter decreases linearly with increasing temperature. In other words, this parameter becomes small as the temperature $T$ approaches Flory's theta temperature. In the previous paper, ${ }^{1}$ we demonstrated for the PS- $\mathrm{CH}$ system that $k$ vanished at Flory's theta temperature $(307 \mathrm{~K})$. For the PS $\mathrm{MCH}$ system, $k$ becomes zero at $c a$. $343 \mathrm{~K}$, which is Flory's theta temperature. ${ }^{8}$ Therefore, it may be concluded that the molecular weight dependence of $\chi$ changes its sign at Flory's theta temperature $\theta$ and that $k$ can be expressed by,

$$
k=k_{0}(1-T / \theta)
$$

$k_{0}$ was found to be 129 for PS-MCH and 108 for PS-CH.

The breadth of the MWD of polymers in the polymer-rich phase expressed in terms of $M_{w} / M_{n}$ is plotted against $\rho_{\mathrm{p}}$ in Figure 6, were open circles are actual experimental data, the broken lines are the theoretical curves at $v_{\mathrm{p}}{ }^{0}=0.5 \times 10^{-2}, 0.86 \times 10^{-2}$, and $2.0 \times 10^{-2}$ for $p=0.7$ and $k=0$ and the full lines are the theoretical relations calculated for $p=0.7$ by taking into consideration the complicated changes in $k$ with phase equilibrium temperature (accordingly $\rho_{\mathrm{p}}$ ) (Figure 5). The experimental $\rho_{\mathrm{p}}$ dependence of $M_{w} / M_{n}$ in the polymer-rich phase can be reasonably interpreted by considering the molecular weight and concentration dependences of the $\chi$ parameter.

\section{REFERENCES}

1. K. Kamide and Y. Miyazaki, Polym. J., 13, 325 (1981).

2. K. Kamide, K. Sugamiya, T. Kawai, and Y. Miyazaki, Polym. J., 12, 67 (1980).

3. K. Kamide, Y. Miyazaki, and T. Abe, Makromol. Chem., 177, 485 (1976).

4. K. Kamide, Y. Miyazaki, and T. Abe, Polym. J., 9, 395 (1977).

5. K. Kamide, K. Sugamiya, T. Ogawa, C. Nakayama, and N. Baba, Makromol. Chem., 135, 23 (1970).

6. K. R. Roberts, J. A. Torkington, M. Gordon, and S. B. Ross-Murphy, J. Polym. Sci., Polym. Symp., No. 61, 45 (1977).

7. W. W. Yau and S. W. Fleming, J. Appl. Polym. Sci., 12, 2111 (1968).

8. T. Dobashi, M. Nakata, and M. Kaneko, Polym. Prepr. Jpn., 27, 1542 (1978).

9. Th. G. Scholte, Eur. Polym. J., 6, 1063 (1970).

10. Th. G. Scholte, J. Polym. Sci., A-2, 9, 1553 (1971). 